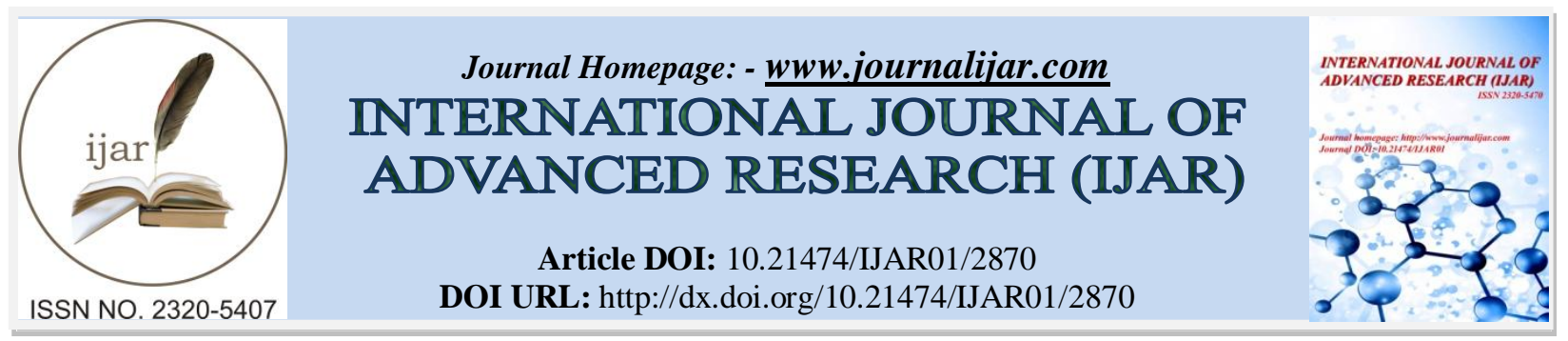

RESEARCH ARTICLE

\title{
ENHANCEMENT OF ENERGY EFFICIENT ROUTING PROTOCOL BY MINIMIZING ENERGY CONSUMPTION IN WIRELESS SENSOR NETWORKS.
}

\author{
Mr. Sharad A. Bhad, Mr. Vikram M. Chavan, Mr. Nilesh S. Nalawade, Mr.Amol P.Nagime. \\ Assistant Professor, Department of E\&TC. Sinhgad Institute Of Technology, Lonavala-410401 Pune, Maharashtra, \\ India.
}

\section{Manuscript Info} [.......................

\section{Manuscript History}

Received: $x x x x x x x x x x x x x x x$ Final Accepted: $x x x x x x x x x x x x$ Published: $\operatorname{xxxxxxxxxxxxxxxx}$

Key words:-

Sleep scheduling routing protocol, Dead node, Energy enhancement, Sink node, Sensor nodes.

\section{Abstract}

A wireless sensor network (WSN) consists of number of sensor nodes along with a sink node that keeps track of all information in the network. These sensor nodes get the power from small some batteries in them. The WSN is used for many applications mostly for the regions that are out reach of human beings e.g. hilly area, mountains, rivers and to get readings of these places to get the environmental changes, we have to connect all nodes, connection of these all nodes forms the graph of the network. There is needed to be synchronized and balanced battery life to increase the life of the network. This is the main motive of our project here we have proposed a protocol which will help us in enlarging the life of network and low battery loss in the network for that we will keep only the sensor nodes that are part of path at the time of transmission and those who are not in use will be kept in sleep mode. This will help us to enhance energy of the battery of and also enhances the energy of entire network.

Copy Right, IJAR, 2016,. All rights reserved.

\section{Introduction:-}

A Wireless Sensor Network (WSN) consists of large numbers of sensor nodes . These sensors are sensing the number of events in the world. All sensor nodes are keeping communication within the nodes by using wireless network only. They are getting power from battery and we know that battery have limited energy. Each sensor node consumes limited power and memory again due to constraints imposed by the available supply of energy. The major function of WSNs is to observe and record events in the environment and report them to the sink .In the process, the sink node also needs to broadcast messages to each node of the WSN, and sensor nodes may need to communicate with each other as well. Wireless sensor network are typically deployed in extreme conditions such as mountainous region, foreign planets such as Moon, Mars etc.

In order to enhance the network life duration, it is very important to minimize the consumption of energy by individual nodes. This would ensure that the connectivity needed to transmit data from a sensor node to sink can always be maintained. And average energy loss per transmission will be minimized.

Another requirement of WSNs for applications such as flood detection, fire detection etc. is that the delay to transmit data from sensor node to the sink should be as minimum as possible. These requirements need to be complete. Completion of requirements is not too easy. The transceiver is the major unit that consumes lots of energy 
in each sensor node even when it is idle. Therefore, sensor nodes are usually put to sleep if they are not required to transmit data or sense environment i.e. minimization of energy consumption and it is really very difficult to integrate sleep scheduling scheme with routing protocols for WSNs Thus we can achieve the objective of routing protocols. We assume that the transceiver, processor, and sensing units can be put to sleep (minimization of energy consumption) independently and when we say that the sensor node is put to sleep, we denote that the transceiver and the processor are put to sleep. The sleep scheduling of sense units can be complete separately to ensure sensing coverage. Here, we propose a fresh sleep scheduling scheme using a tree, and an energy aware routing protocol that is appropriately integrated with the above sleep scheduling scheme with a view to meet the objectives for routing protocols as given above.

\section{Project scope:-}

The energy routing protocol in this project is intended for WSNs in which sensor nodes are static. Besides the applications running in the WSN require that the information's gathered by the sensor nodes have to be transmitted quickly to the sink. We are assuming that each node has a unique ID, and the communication between neighboring nodes is symmetric and bidirectional. It is as well unspecified that the clocks of the sensor nodes in the WSN are synchronized so that nodes can be woken up nearly at the same time i.e. synchronization and they can execute the protocol. The objectives of energy routing protocol with sleep scheduling are as follows.

1. Many sensor nodes should be asleep many of the time so that the energy consumption per node is reduced.

2. Consumption of energy by all the sensor nodes of systems remains balanced, i.e., at any time, consumption of energy of each node should be same.

3. Transmission time of data from a sensor node to the sink is as minimum as possible as i.e. energy consumption will be minimum.

\section{Project Architecture:-}

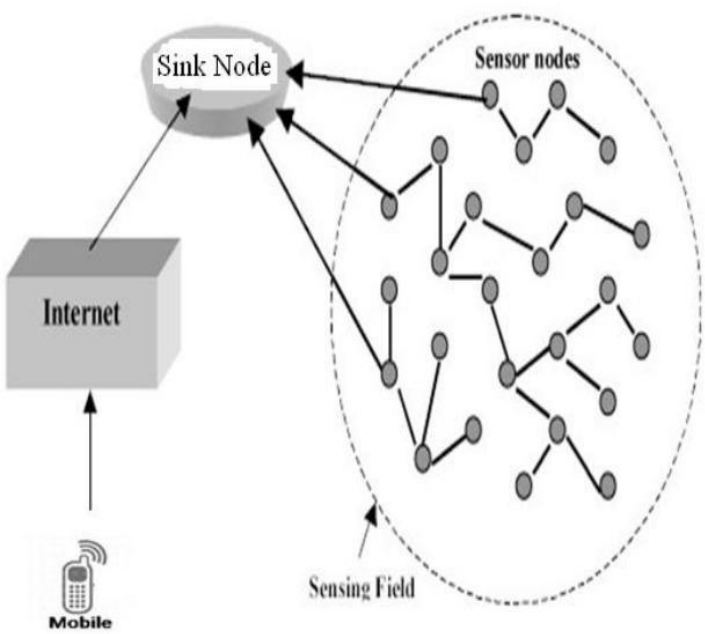

Fig 1:- Project architecture

Energy routing protocol with sleep scheduling. The energy routing protocol is intended for WSNs in which sensor nodes are static. Besides the applications running in the WSN require that the information shared by the sensor nodes from the sensing field have to be transmitted urgently to the sink. Next, we have to consider that each node has a unique ID, and the communication between neighboring nodes is symmetric and bidirectional. It is as well unspecified that the clocks of the sensor nodes of all in the WSN are synchronized so that nodes can be woken up nearly at the same time, and they can execute the energy routing protocol.

Also with the special module that will inform the administrator about any attack that has appeared at any of the nodes in our wireless sensor network by sending the details of the attack in the form of an message notification to his mobile phone. 


\section{Overview of energy routing protocol:-}

System is divided in two parts 1.Sensor node \& 2. Sink node. Sensor node is one of the nodes in the network except the one who receives data sensor node's work is to provide the data to be delivered to the sink node and the information about its neighbors along with their battery lives. Sink node find its the path of data delivery and also one who receives the data from the sensor nodes.

We plot a graph of network that we have to deal with the entire sensor node who is the information sender then. Sink node divides the sensor node in internal nodes and Leaf nodes basically internal nodes are those who have active participation in the data delivery and leaf nodes are those whom we don't need during current data delivery. And according to our protocol we keep the internal nodes awaken and let the leaves sleep till this data transmission is completed. Administrator is informed if any node is under attack. Thus we did the energy consumption by doing the sleeping the nodes whenever not required and wake up the sensors whenever required. Also we made the equal energy consumption of all sensors nodes and proper synchronization.

\section{Results of energy routing protocol:-}

We have obtained few results by comparing our system with few of the previously existing systems. Following are the results by comparison with the GSP [4].

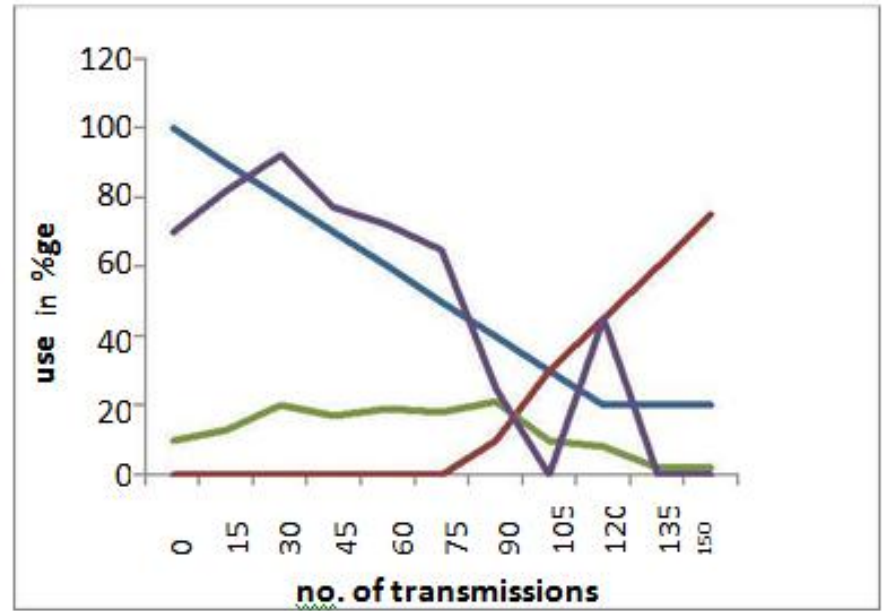

Fig 2:- Percentage of active nodes and dead nodes, packets delivered, and average remaining energy in unipath approach.

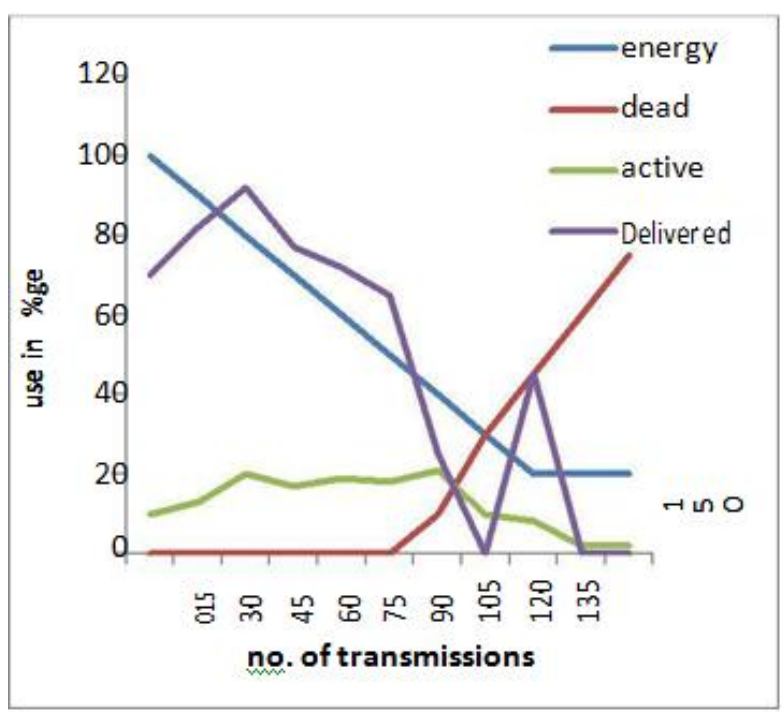

Fig 3:- Percentage of active nodes and dead nodes, packets delivered, and average remaining energy in our approach. 
Here the two graphs look same but our protocol is different from the GSP [4]. Because our protocol takes very less time than it, reason behind that is table that consists of the energy value of each node and it is automatically updated after each transmission is complete. While in GSP [4] the table is created each time while we are going to transmit other node or the same node.

Following figure shows the comparison of our protocol with the traditional approaches that were used previously even before the GSP [4]. From the following graph it is easy to predict how energy efficient our protocol is, e.g. if we consider the total battery consumption in the network in 8 transmissions by taking integer value $60 /$ transmission then if the network is of 8 nodes the battery consumption will exceed integer value 3500 , but using our proposed protocol the value is even less than integer value 2000 . The result says it all.

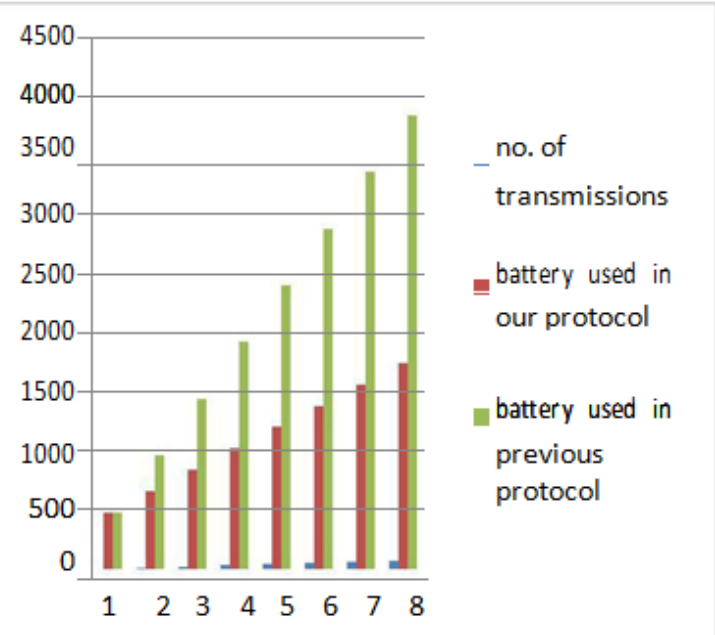

Fig. 4:- Comparison of battery use in our protocol \& previo us protocol.

\section{Conclusion:-}

We presented an energy aware routing protocol with sleep scheduling for WSNs. The core of the routing protocol is the efficient construction of the broadcast tree with two paths from each node towards the sink, and with higher remaining energy at each internal node of the tree. The tree is reconstructed at the beginning of each period so that none of these nodes dies before other nodes, which means that all nodes will die at around the same time. Consecutive packets are routed through alternative path to reduce traffic in individual paths. Leaf node sleep mechanism is highly energy efficient as more number of nodes is able to sleep, and this helps to prolong the network lifetime.

\section{References:-}

1. R. Mitra and D. Nandy. A survey on clustering techniques for wireless sensor network. Int. J. of Research in Computer Science, 2(4):51\{57, 2012.

2. S.S. Kanhere N. Ahmed and S. Jha. The holes problem in wireless sensor networks:a survey. ACM SIGMOBILE Mobile Computing and Communications Review, 9(2):4\{18, April 2005. URL doi:10.1145/1072989.1072992.

3. N. Chand V. Katiyar and S. Soni. A survey on clustering algorithms for heterogeneous wireless sensor networks. Int. J. Advanced Networking and Applications, 2(4):745\{754, 2011.

4. S. Jabbehdari R. Sheikhpour and A. Khadem-Zadeh. Comparison of energy efficient clustering protocols in heterogeneous wireless sensor networks. InternationalJournal of Advanced Science and Technology, 36:27\{40, November 2011

5. K. Akkaya and M. Younis. A survey on routing protocols for wireless sensor networks, ad hoc networks. Ad Hoc Networks, CiteSeerX, 3:325 \{349, 2005.URLhttp://citeseer.ist.psu.edu/viewdoc/summa ry?doi=10.1.1.85.3616.

6. An energy Efficient routing protocol with sleep scheduling for wireless sensor networks. By Amulya Ratna Swain, R. C. Hansdah and Vinod Kumar Chouhan Department of Computer Science and Automation Indian Institute of Science Bangalore, India

7. J. L. Hill. System architecture for wireless sensor networks. Ph.D. dissertation,University of California atBerkeley,Spring,2003. 